Supporting Information

\title{
Electrostatically Fabricated Three-Dimensional Magnetite and MXene Hierarchical Architecture for Advanced Lithium-Ion Capacitors
}

Shijie Wang, ${ }^{\dagger}$ Dongdong Jin, ${ }^{\dagger}$ Ye Bian, ${ }^{\ddagger}$ Rutao Wang, ${ }^{*},, \dagger$ and Li Zhang $*,+$

* Department of Mechanical and Automation Engineering, The Chinese University of Hong Kong,

Shatin, N.T., Hong Kong SAR, 999077, China.

$\S$ Key Laboratory for Liquid-Solid Structural Evolution and Processing of Materials, Ministry of Education, School of Materials Science and Engineering, Shandong University, Jinan, 250061, Shandong, China.

$†$ Suzhou Institute of Shandong University, Suzhou, 215123, Jiangsu, China

* Corresponding Author E-mails: lizhang@mae.cuhk.edu.hk (L.Z.); rtwang@sdu.edu.cn (R.W). 


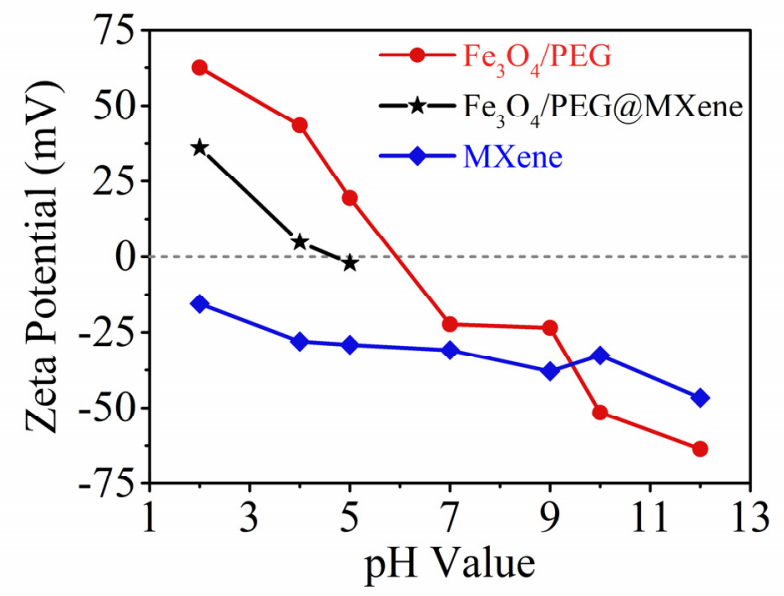

Figure S1. Zeta potential of individual $\mathrm{Fe}_{3} \mathrm{O}_{4} / \mathrm{PEG}$ and MXene, as well as self-assembled $\mathrm{Fe}_{3} \mathrm{O}_{4} / \mathrm{PEG} @ \mathrm{MXene}$ at different $\mathrm{pH}$ values.

The intrinsic negative surface charge of MXene is derived from hydroxyl, oxygen, and fluorine terminations, which is relatively stable at acidic to basic environment. ${ }^{1,2}$ Instead, the surface charge of $\mathrm{Fe}_{3} \mathrm{O}_{4} / \mathrm{PEG}$ is $\mathrm{pH}$ dependent, which is probably related to the transformation of surface iron complexes. ${ }^{3}$ In acidic solution, $\mathrm{Fe}(\mathrm{II}, \mathrm{III}) \mathrm{OH}_{2}{ }^{+}$dominates and shows a significant positive surface charge. When $\mathrm{pH}$ is increased gradually, $\mathrm{Fe}(\mathrm{II}, \mathrm{III}) \mathrm{OH}_{2}{ }^{+}$transforms to $\mathrm{Fe}(\mathrm{II}, \mathrm{III}) \mathrm{OH}$ and Fe(II,III)Osequentially, leading to quasi-neutral and negative surface charge, respectively. Considering such $\mathrm{H}^{+}$-induced surface charge mechanism (i.e., add or remove $\mathrm{H}^{+}$to/from the surface), the surface charge of $\mathrm{Fe}_{3} \mathrm{O}_{4} / \mathrm{PEG}$ can be therefore treated as quasi-intrinsic, which is different from the extrinsic counterpart derived from surface modifications. After electrostatic self-assembly to $\mathrm{Fe}_{3} \mathrm{O}_{4} / \mathrm{PEG} @$ MXene nanocomposites, partial neutralization of zeta potential can be observed, indicating the effectiveness of such strategy. 

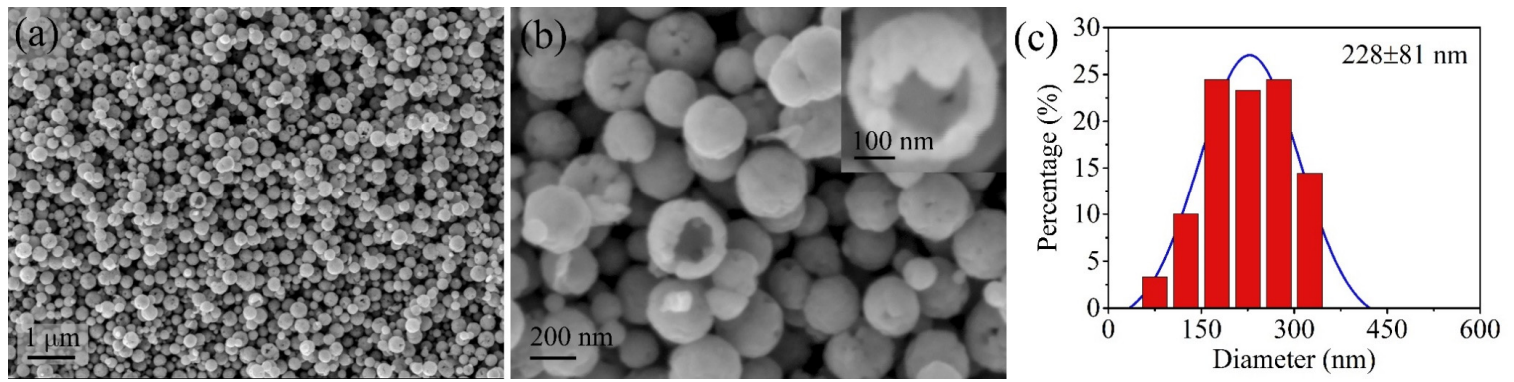

Figure S2. SEM images and size distribution of hollow $\mathrm{Fe}_{3} \mathrm{O}_{4} / \mathrm{C}$ microspheres. (a) Lowmagnification SEM image. (b) High-magnification SEM image. Inset shows the typical inner void space from a cracked microsphere. (c) Size distribution measured from (b).
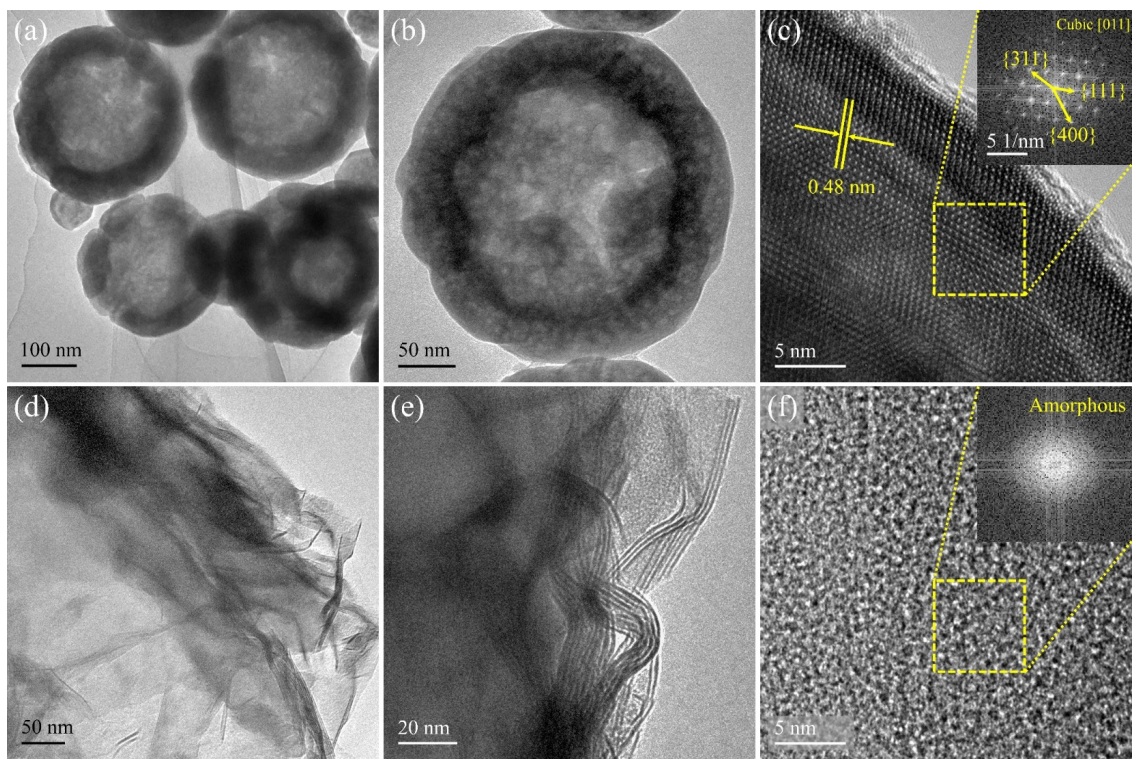

Figure S3. TEM images of individual $\mathrm{Fe}_{3} \mathrm{O}_{4} / \mathrm{C}$ and MXene. (a-b) TEM images of hollow $\mathrm{Fe}_{3} \mathrm{O}_{4} / \mathrm{C}$ microspheres. (c) HRTEM image of $\mathrm{Fe}_{3} \mathrm{O}_{4} / \mathrm{C}$. Inset is the reduced FFT image of the selected area showing a [011] crystalline zone. (d-e) TEM images of the few-layer MXenes. (f) HRTEM image of MXene. Inset is the reduced FFT image of the selected area showing the amorphous characteristic. 


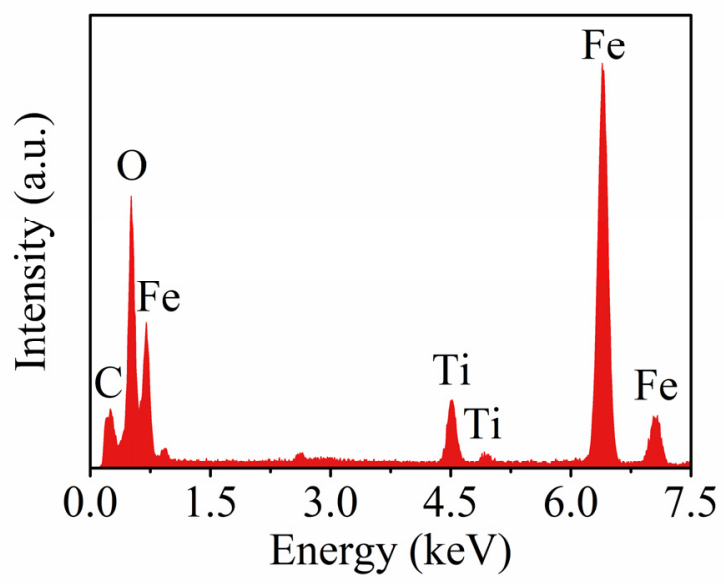

Figure S4. Representative EDS spectrum of $\mathrm{Fe}_{3} \mathrm{O}_{4} / \mathrm{C} @ M X e n e$ nanocomposites.
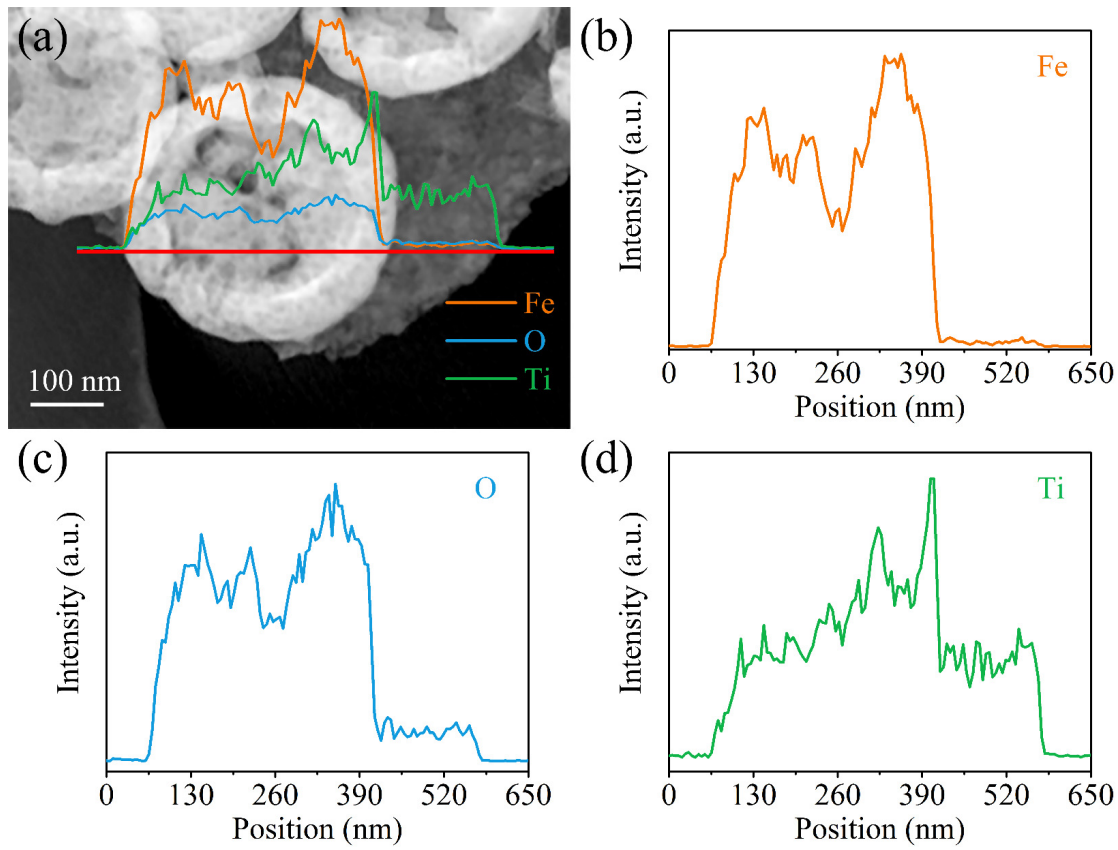

Figure S5. EDS linear scan on $\mathrm{Fe}_{3} \mathrm{O}_{4} / \mathrm{C} @ M X$ Xene nanocomposites. (a) STEM image, with red horizontal line indicating the path of linear scan. Received characteristic X-ray signals of Fe, O, and Ti elements are overlapped in the image. (b-d) Linear distribution of $\mathrm{Fe}, \mathrm{O}$, and Ti elements along the scanned path. 


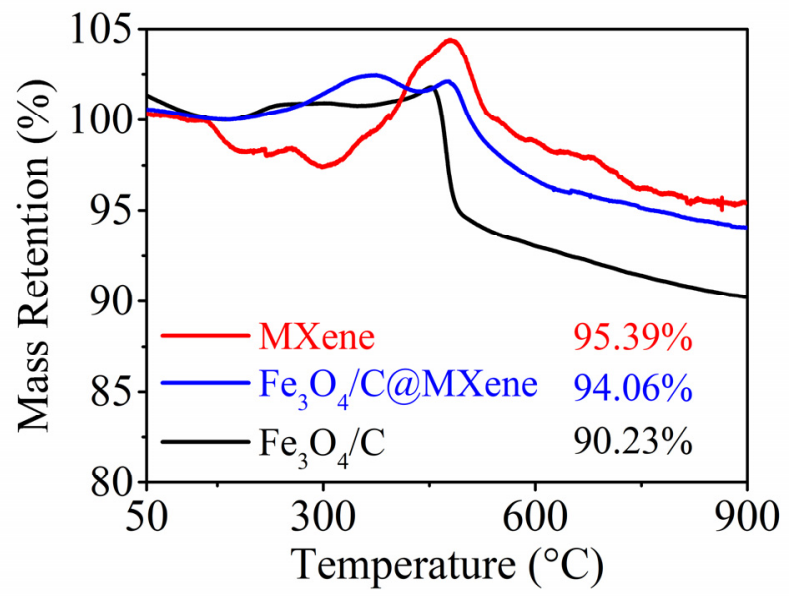

Figure S6. TGA profile of pristine $\mathrm{Fe}_{3} \mathrm{O}_{4} / \mathrm{C}$, MXene, and self-assembled $\mathrm{Fe}_{3} \mathrm{O}_{4} / \mathrm{C} @$ MXene in $\mathrm{O}_{2}$ atmosphere.

The following chemical reactions in $\mathrm{O}_{2}$ occur during complete annealing:

$$
\begin{gathered}
4 \mathrm{Fe}_{3} \mathrm{O}_{4}+\mathrm{O}_{2} \stackrel{\Delta}{\longrightarrow} 6 \mathrm{Fe}_{2} \mathrm{O}_{3} \\
\mathrm{C}+\mathrm{O}_{2} \stackrel{\Delta}{\longrightarrow} \mathrm{CO}_{2} \\
\mathrm{Ti}_{3} \mathrm{C}_{2}+5 \mathrm{O}_{2} \stackrel{\Delta}{\longrightarrow} 3 \mathrm{TiO}_{2}+2 \mathrm{CO}_{2}
\end{gathered}
$$

For $\mathrm{Fe}_{3} \mathrm{O}_{4} / \mathrm{C}$, the 9.77 wt.\% mass reduction is a synergistic effect of $\mathrm{Fe}_{3} \mathrm{O}_{4}$ oxidation and carbon combustion. The original carbon content is estimated to be $12.8 \mathrm{wt} . \%$. Similarly, for pure $\mathrm{Ti}_{3} \mathrm{C}_{2} \mathrm{~T}_{\mathrm{x}}$ MXene ( $\mathrm{T}_{\mathrm{x}}$ represents the -OH, -O-, and -F terminating functional groups), the mass loss of 4.61 wt.\% originates from both $\mathrm{Ti}_{3} \mathrm{C}_{2}$ oxidation and $\mathrm{T}_{\mathrm{x}}$ removal. $\mathrm{The} \mathrm{Ti}_{3} \mathrm{C}_{2}$ content is estimated to be 66.7 wt.\%. The mass retention of $\mathrm{Fe}_{3} \mathrm{O}_{4} / \mathrm{C} @ M X e n e$ is between that of $\mathrm{Fe}_{3} \mathrm{O}_{4} / \mathrm{C}$ and MXene, yet the estimation of the content of both components induces large error since a mass retention difference of "95.39 $-90.23=5.16$ wt.\%" determines the $0-100$ wt.\% of $\mathrm{Fe}_{3} \mathrm{O}_{4} / \mathrm{C}$ or MXene. 


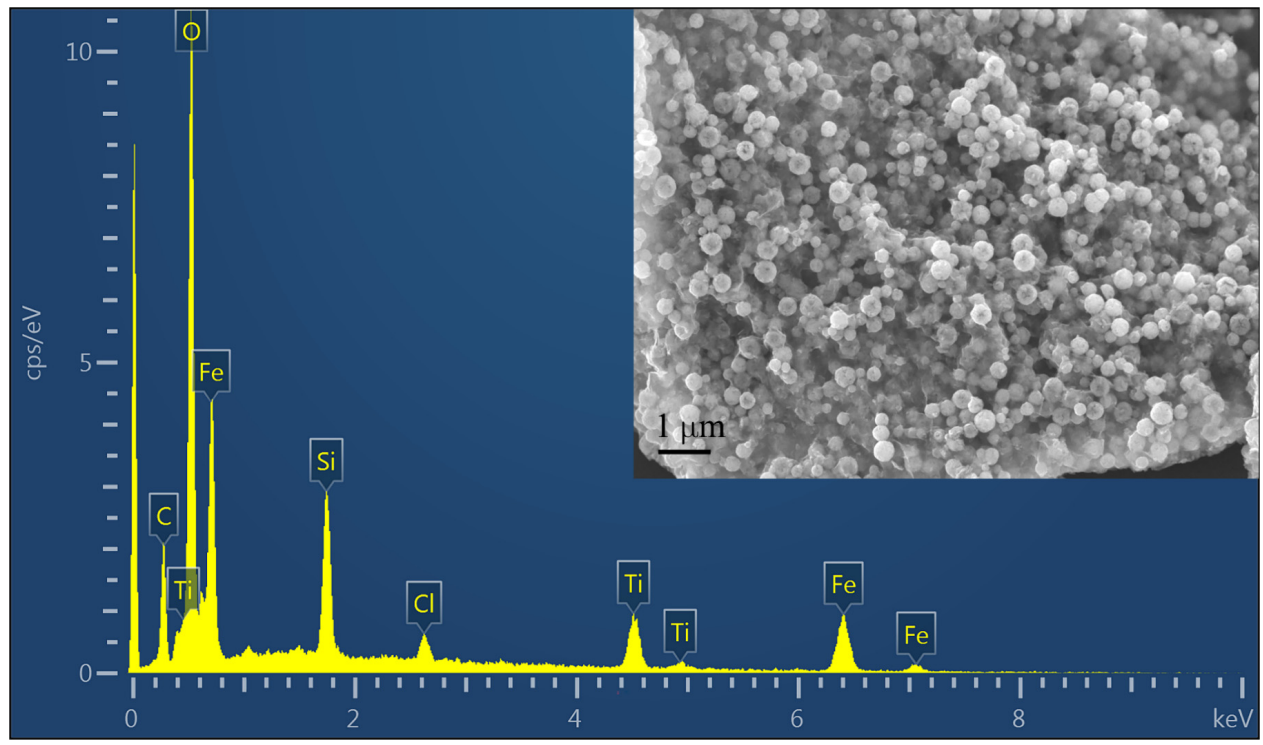

Figure S7. EDS areal scan on $\mathrm{Fe}_{3} \mathrm{O}_{4} / \mathrm{C} @$ MXene nanocomposites under SEM. Inset is the SEM image showing the selected area for elemental analysis.

Table S1. Mass content of major elements in $\mathrm{Fe}_{3} \mathrm{O}_{4} / \mathrm{C} @ \mathrm{MX}$-ne from the EDS spectrum in Figure $\mathrm{S} 7$. Signals from substrate $\mathrm{Si}$ and minor impurities $(\mathrm{Cl})$ are ignored.

\begin{tabular}{ccc}
\hline Element & Mass Content (wt.\%) & Mass Ratio \\
\hline Iron $(\mathrm{Fe})$ & 52.65 & 4.37 \\
Titanium $(\mathrm{Ti})$ & 12.05 & 1.00 \\
Oxygen $(\mathrm{O})$ & 26.60 & $/$ \\
Carbon $(\mathrm{C})$ & 8.71 & $/$ \\
\hline
\end{tabular}

For EDS characterization, the mass contents of elements are semi-quantitative. Specifically, the mass content of light elements (i.e., atomic number $<10$ ) are not accurate. Hence, only the mass ratio of $\mathrm{Fe}$ and $\mathrm{Ti}$ are adopted for calculation, which is supplemented by the TGA results that 12.8 wt.\% carbon exists in $\mathrm{Fe}_{3} \mathrm{O}_{4} / \mathrm{C}$ and 66.7 wt.\% $\mathrm{Ti}_{3} \mathrm{C}_{2}$ exists in MXene. Calculated mass content of $\mathrm{Fe}_{3} \mathrm{O}_{4}$, carbon, and MXene are 69.6 wt.\%, 10.2 wt.\%, and 20.2 wt.\%, respectively. 
(a)

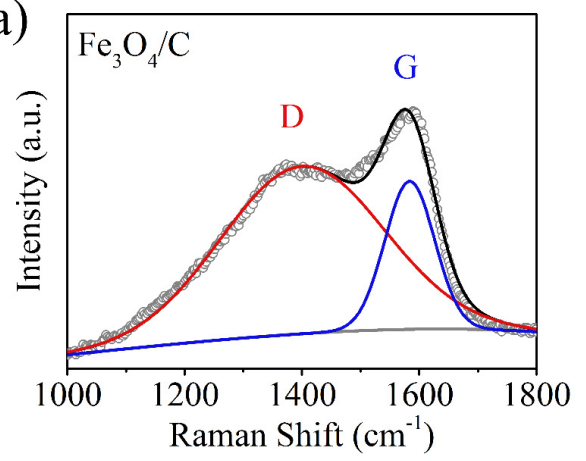

(b)

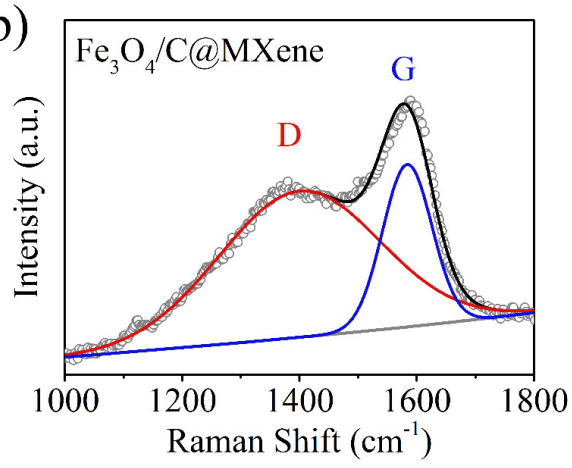

Figure S8. Raman spectrum of (a) $\mathrm{Fe}_{3} \mathrm{O}_{4} / \mathrm{C}$ and (b) $\mathrm{Fe}_{3} \mathrm{O}_{4} / \mathrm{C} @ M X$ Xene showing the characteristic D- and G-band of carbon.

Two broad peaks at 1400 and $1584 \mathrm{~cm}^{-1}$ in Figure S8 can be assigned to D- and G-band of carbon, respectively. D-band represents the $\mathrm{A}_{1 \mathrm{~g}}$ vibration mode of $\mathrm{sp}^{3}$-bonded carbon at the edge of a graphene layer, while G-band represents the $\mathrm{E}_{2 \mathrm{~g}}$ vibration mode in $\mathrm{sp}^{2}$-bonded graphitic carbon. ${ }^{4,5}$ Specifically, the $I_{D}: I_{G}$ value of $\mathrm{Fe}_{3} \mathrm{O}_{4} / \mathrm{C}$ and $\mathrm{Fe}_{3} \mathrm{O}_{4} / \mathrm{C} @ M X e n e$ is 3.70 and 2.92 , respectively. The in-plane crystallite size $\left(L_{a}, \mathrm{~nm}\right)$ can thus be obtained by Eq. (S4): ${ }^{6}$

$$
L_{a}=\frac{560}{E_{l}^{4}} \times\left(\frac{I_{D}}{I_{G}}\right)^{-1}
$$

where $E_{l}(\mathrm{eV})$ is excitation laser energy, i.e., $2.41 \mathrm{eV}$ for incident laser $\lambda=514 \mathrm{~nm}$. Calculated average domain sizes are 4.49 and $5.69 \mathrm{~nm}$, respectively, suggesting disordered nature of carbon within the $\mathrm{Fe}_{3} \mathrm{O}_{4} / \mathrm{C}$ nanostructure. 


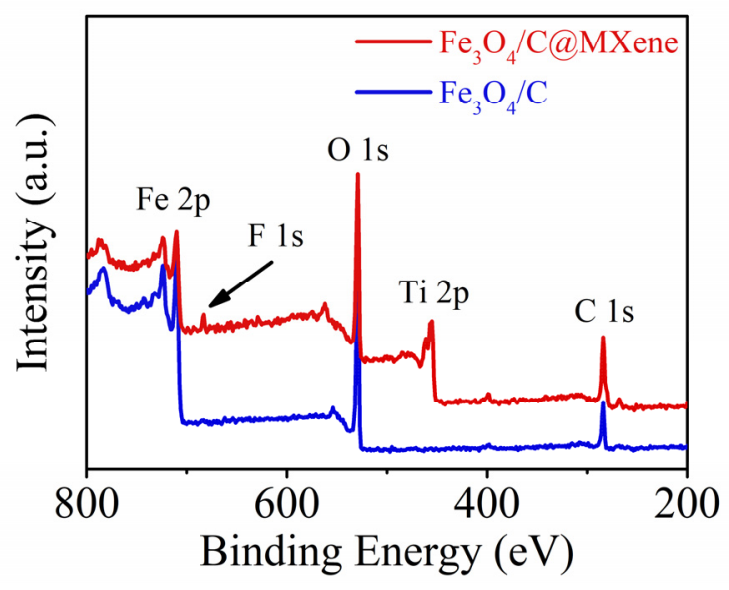

Figure S9. XPS survey spectra of $\mathrm{Fe}_{3} \mathrm{O}_{4} / \mathrm{C}$ and $\mathrm{Fe}_{3} \mathrm{O}_{4} / \mathrm{C} @ M X e n e$. Fe 2p, Ti 2p, O 1s, and C 1s peaks are significant, while $\mathrm{F}$ 1s peak is marginally observable.
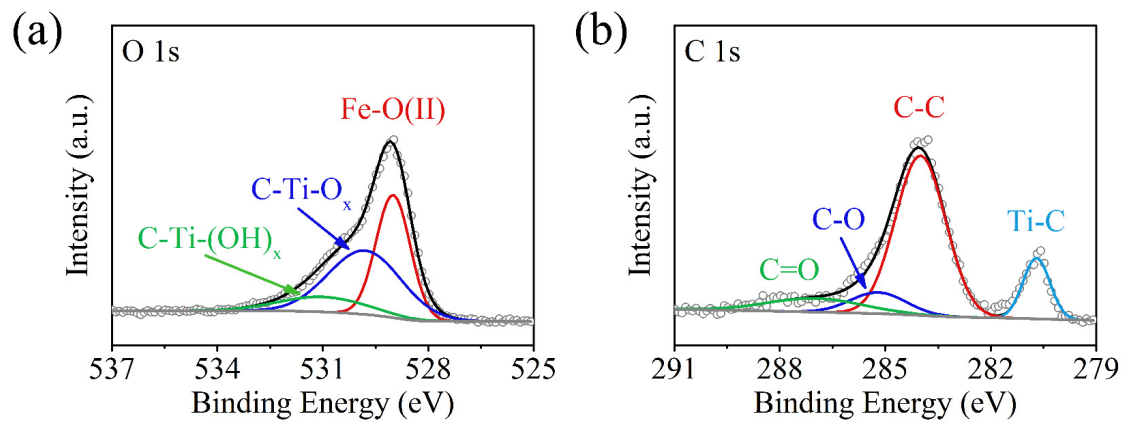

Figure S10. XPS orbit spectra of $\mathrm{Fe}_{3} \mathrm{O}_{4} / \mathrm{C} @$ MXene: (a) O 1s; (b) C 1s.
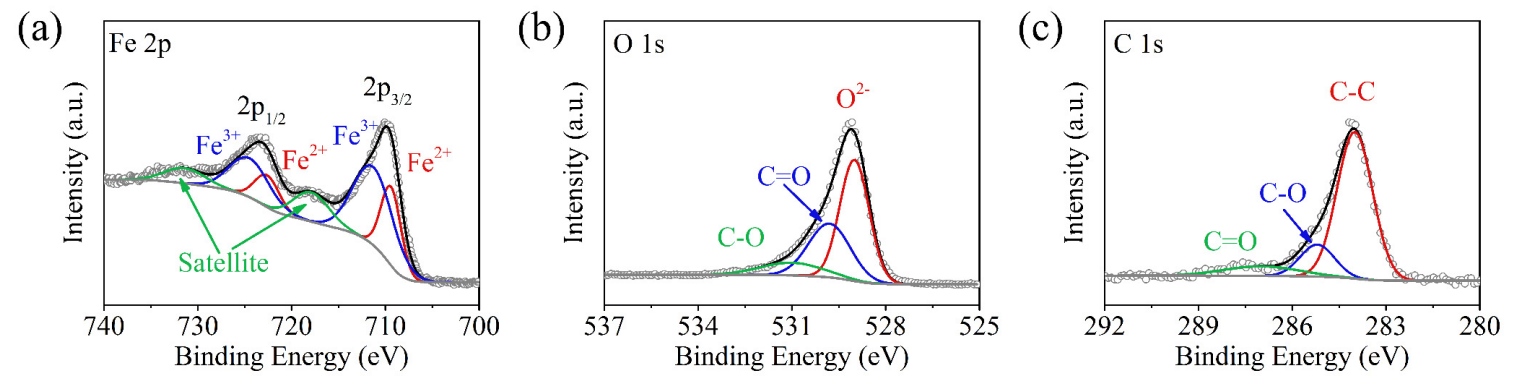

Figure S11. XPS orbit spectra of $\mathrm{Fe}_{3} \mathrm{O}_{4} / \mathrm{C}$ : (a) Fe 2p; (b) O 1s; (c) C 1s. 


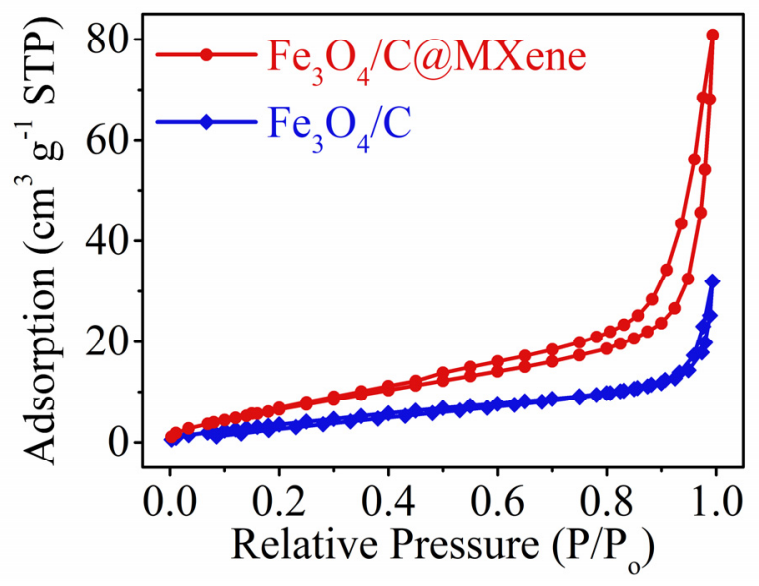

Figure S12. $\mathrm{N}_{2}$ adsorption and desorption isotherms of $\mathrm{Fe}_{3} \mathrm{O}_{4} / \mathrm{C}$ and $\mathrm{Fe}_{3} \mathrm{O}_{4} / \mathrm{C} @ \mathrm{MXene}$. 


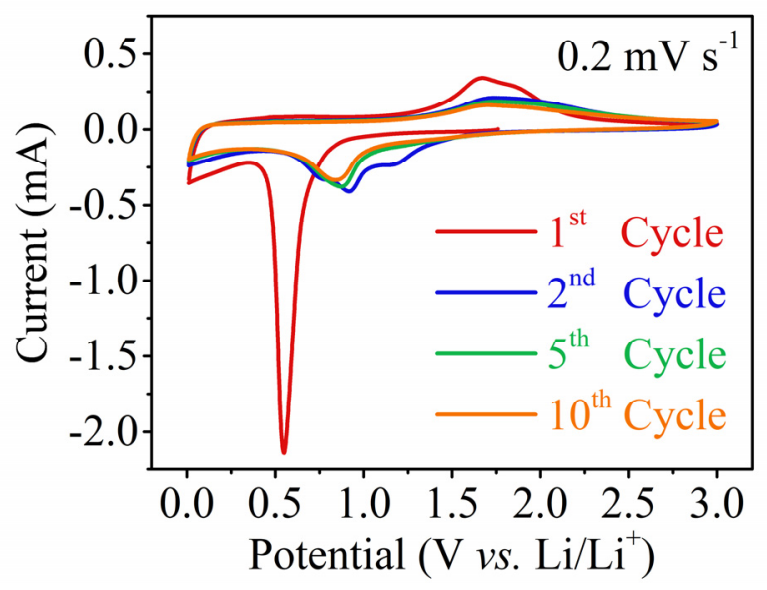

Figure S13. Initial cycles of $\mathrm{CV}$ curves of $\mathrm{Fe}_{3} \mathrm{O}_{4} / \mathrm{C} @ \mathrm{MXene}$ at $0.2 \mathrm{mV} \mathrm{s}^{-1}$ in half-cell configuration $\left(0.01-3.0 \mathrm{~V} v s . \mathrm{Li} / \mathrm{Li}^{+}\right)$. 
(a)

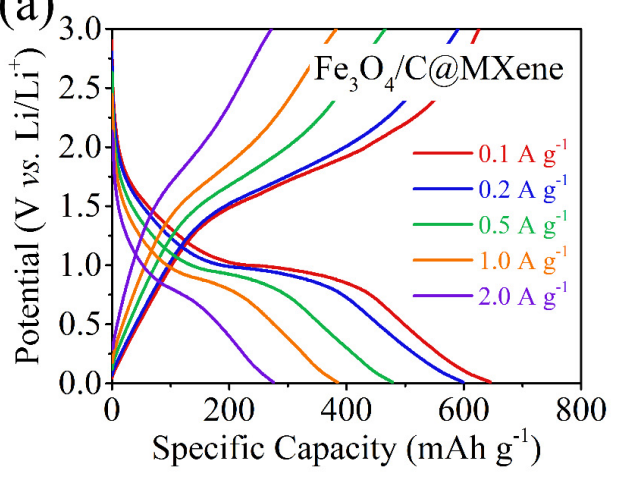

(c)

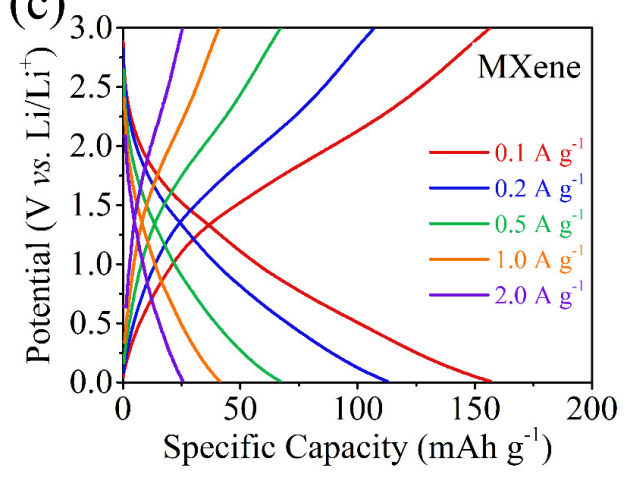

(b)

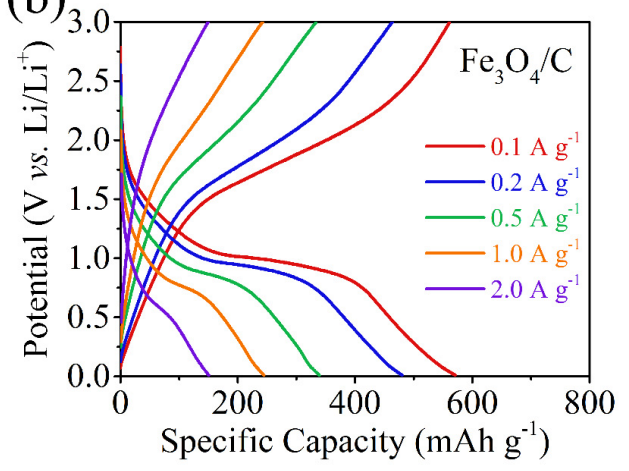

(d)

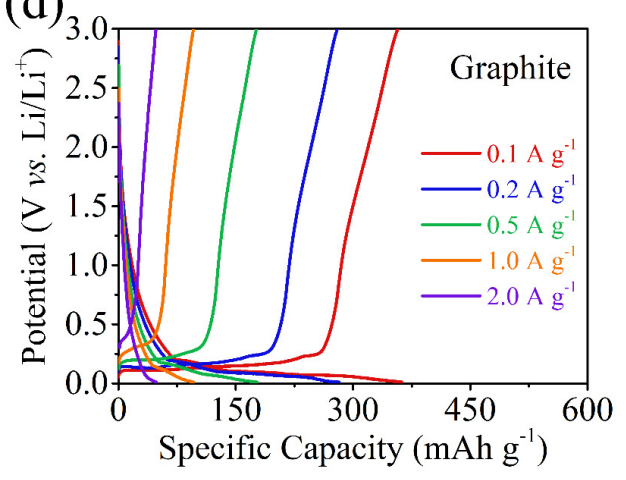

Figure S14. GCD curves of different anode materials in half-cell configuration (0.01-3.0 V vs.

$\mathrm{Li} / \mathrm{Li}^{+}$): (a) $\mathrm{Fe}_{3} \mathrm{O}_{4} / \mathrm{C} @ M X e n e ; ~(b) ~ \mathrm{Fe}_{3} \mathrm{O}_{4} / \mathrm{C}$; (c) MXene; (d) Graphite.

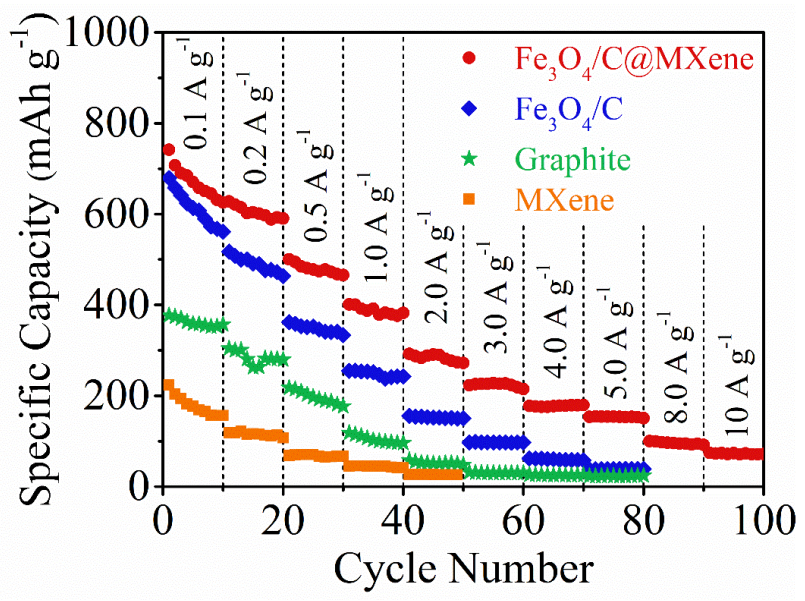

Figure S15. Specific capacity of different anode materials in half-cell configuration (0.01-3.0 V $\left.v s . \mathrm{Li} / \mathrm{Li}^{+}\right)$at a variety of current densities. 
Table S2. Comparison of electrochemical performance among different anode materials.

\begin{tabular}{|c|c|c|c|c|c|}
\hline \multirow[b]{2}{*}{ Materials } & \multirow{2}{*}{$\begin{array}{c}\text { Working } \\
\text { Potential } \\
(\mathbf{V} v s . \\
\left.\mathbf{L i} / \mathbf{L i}^{+}\right)\end{array}$} & \multicolumn{2}{|c|}{ Best Performance } & \multicolumn{2}{|c|}{ Rate Capability } \\
\hline & & $\begin{array}{l}\text { Specific } \\
\text { Capacity } \\
\left(\mathrm{mAh} \mathrm{g}^{-1}\right)\end{array}$ & $\begin{array}{l}\text { Current } \\
\text { Density } \\
\left(\mathrm{A} \mathrm{g} \mathrm{g}^{-1}\right)\end{array}$ & $\begin{array}{l}\text { Specific } \\
\text { Capacity } \\
\left(\mathbf{m A h} \mathbf{g}^{-1}\right)\end{array}$ & $\begin{array}{c}\text { Current } \\
\text { Density } \\
\left(\mathbf{A} \mathbf{g}^{-1}\right)\end{array}$ \\
\hline $\mathrm{Fe}_{3} \mathrm{O}_{4} @ \mathrm{MXene}^{7}$ & $0.01-3.0$ & 710 & 0.03 & 100 & 3.0 \\
\hline $\mathrm{Fe}_{3} \mathrm{O}_{4} @ M X e n e @ \mathrm{C}^{8}$ & $0.01-3.0$ & 518 & 0.08 & 242 & 2.0 \\
\hline $\mathrm{Fe}_{3} \mathrm{O}_{4} @ \mathrm{MXene}^{9}$ & $0.01-3.0$ & 417 & 0.13 & 153 & 2.5 \\
\hline $\mathrm{Fe}_{3} \mathrm{~S}_{4} @ \mathrm{rGO}^{10}$ & $0.01-3.0$ & 614 & 0.10 & 297 & 2.0 \\
\hline $\mathrm{TiO}_{2} / \mathrm{Fe}_{3} \mathrm{O}_{4} @ \mathrm{rGO}^{11}$ & $0.01-3.0$ & 329 & 1.00 & 169 & 8.0 \\
\hline $\mathrm{Co}_{3} \mathrm{O}_{4} @ \mathrm{MXene}^{12}$ & $0.01-3.0$ & 800 & 0.16 & 50 & 6.4 \\
\hline $\mathrm{TiO}_{2} @ \mathrm{MXene}^{13}$ & $0.01-3.0$ & 272 & 0.05 & 140 & 2.0 \\
\hline rGO-MXene ${ }^{14}$ & $0.01-3.0$ & 336 & 0.05 & 101 & 4.0 \\
\hline $\mathrm{MoS}_{2} @ \mathrm{MXene}^{15}$ & $0.01-3.0$ & 548 & 0.05 & 90 & 5.0 \\
\hline $\begin{array}{c}\mathrm{Fe}_{3} \mathrm{O}_{4} / \mathrm{C} \\
\text { (This Work) }\end{array}$ & 0.01-3.0 & 561 & 0.10 & 39 & 5.0 \\
\hline $\begin{array}{c}\mathrm{Fe}_{3} \mathrm{O}_{4} / \mathrm{C} @ M X e n e \\
\text { (This Work) }\end{array}$ & 0.01-3.0 & 625 & 0.10 & 72 & 10.0 \\
\hline
\end{tabular}




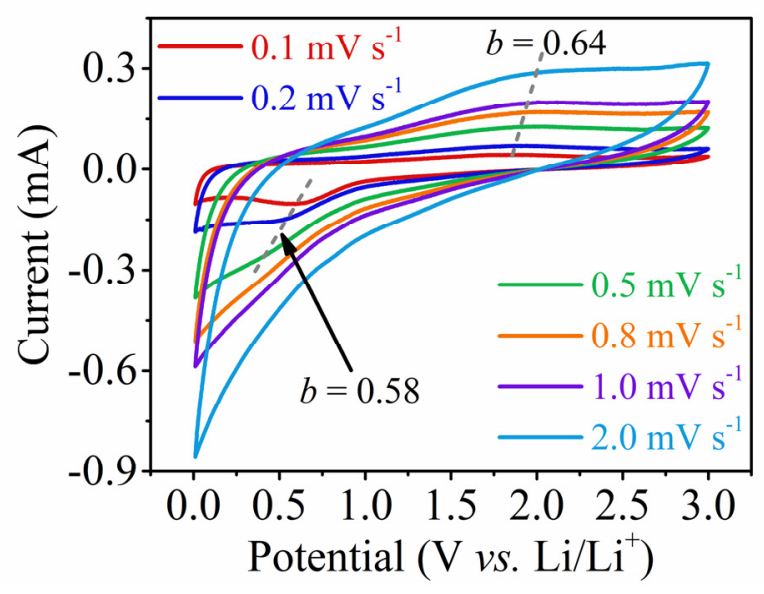

Figure S16. CV curves of $\mathrm{Fe}_{3} \mathrm{O}_{4} / \mathrm{C}$ in half-cell configuration $\left(0.01-3.0 \mathrm{~V} v s . \mathrm{Li} / \mathrm{Li}^{+}\right)$at various of voltage scan rates from 0.1 to $2 \mathrm{mV} \mathrm{s}^{-1}$. Dashed lines mark the positions of anodic and cathodic peak currents, at which $b$ values are calculated. Cathodic polarization problem is severe, so that $b$ value is only fitted up to $0.5 \mathrm{mV} \mathrm{s}^{-1}$.
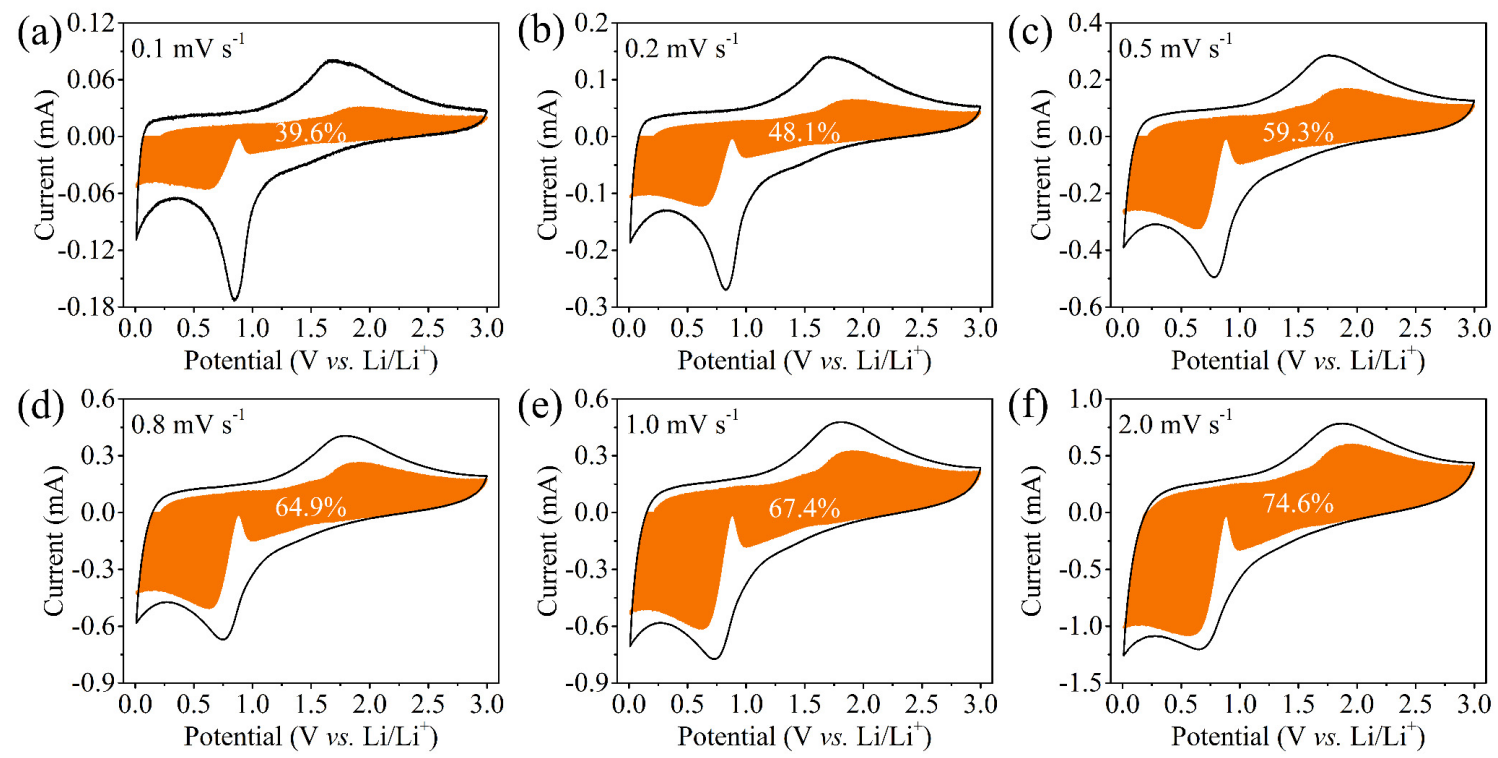

Figure S17. Capacitive current contribution of $\mathrm{Fe}_{3} \mathrm{O}_{4} / \mathrm{C} @$ MXene at different voltage scan rates in half-cell configuration (0.01-3.0 V vs. Li/Li $\left.{ }^{+}\right)$: (a) $0.1 \mathrm{mV} \mathrm{s}^{-1}$; (b) $0.2 \mathrm{mV} \mathrm{s}^{-1}$; (c) $0.5 \mathrm{mV} \mathrm{s}^{-1}$; (d) $0.8 \mathrm{mV} \mathrm{s}^{-1}$; (e) $1.0 \mathrm{mV} \mathrm{s}^{-1}$; (f) $2.0 \mathrm{mV} \mathrm{s}^{-1}$. 

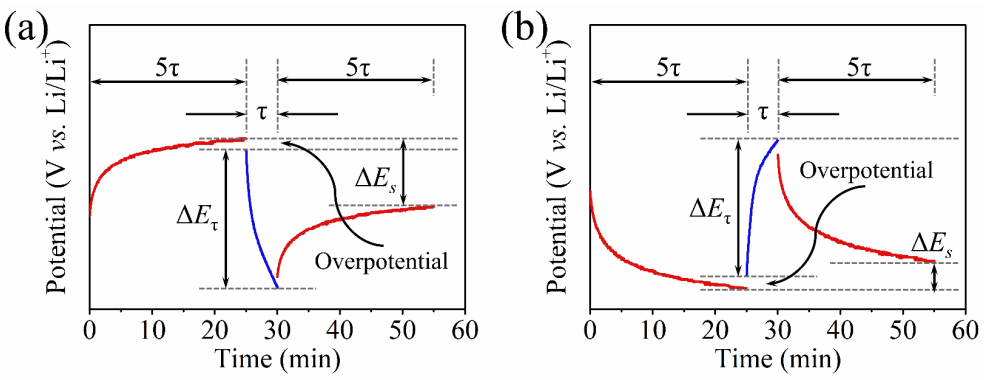

Figure S18. Schematic illustration of essential parameters in GITT analysis during (a) discharging and (b) charging process.
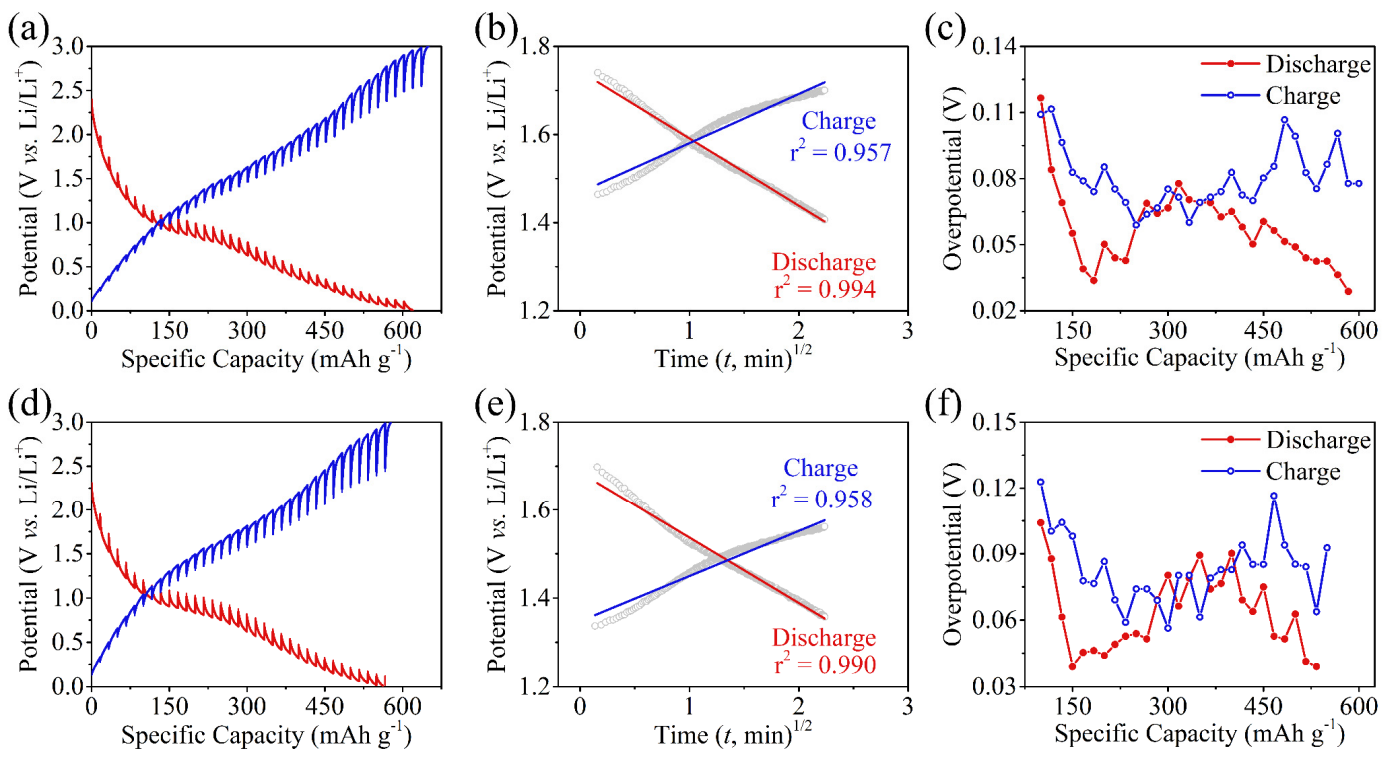

Figure S19. GITT Characterization. (a) GITT potential profile of $\mathrm{Fe}_{3} \mathrm{O}_{4} / \mathrm{C} @ M X e n e$. (b) Potential variation during charge and discharge at around mid-potential (ca. $\left.1.5 \mathrm{~V} v s . \mathrm{Li} / \mathrm{Li}^{+}\right)$as a demonstration of the linear relationship. (c) Overpotential at different states of charge/discharge. (d) GITT potential profile of $\mathrm{Fe}_{3} \mathrm{O}_{4} / \mathrm{C}$. (e) Potential variation during charge and discharge at around mid-potential (ca. $\left.1.5 \mathrm{~V} v s . \mathrm{Li} / \mathrm{Li}^{+}\right)$as a demonstration of the linear relationship. (f) Overpotential at different states of charge/discharge. 
GITT is an effective strategy to estimate the apparent $\mathrm{Li}^{+}$ion diffusion coefficient at different quasi-equilibrium potentials. During the test, a relatively small titration current density (i.e., $0.2 \mathrm{~A}$ $\left.\mathrm{g}^{-1}\right)$ is applied for a relatively short period (i.e., $\left.\tau=5 \mathrm{~min}=300 \mathrm{~s}\right)$ to induce a potential $\operatorname{shift}\left(\Delta E_{\tau}\right)$, followed by much longer relaxation period (i.e., $5 \tau=25 \mathrm{~min}=1500 \mathrm{~s}$ ) to reach a quasi-equilibrium potential for the calculation of $\Delta E_{s}$ (Figure S18). The above titration-relaxation cycle is performed continuously at the whole potential window (i.e., 0.01-3.0 V vs. $\mathrm{Li}^{-\mathrm{Li}^{+}}$) to give a completed potential profile (Figure S19a and S19d).

The apparent $\mathrm{Li}^{+}$diffusion coefficient $\left(D, \mathrm{~cm}^{2} \mathrm{~s}^{-1}\right)$ is calculated based on the following equation with sufficiently small current: ${ }^{16-18}$

$$
D=\frac{4}{\pi}\left(\frac{m V_{m}}{M A}\right)^{2}\left(\frac{\Delta E_{s} / \tau}{d E_{\tau} / d \sqrt{\tau}}\right)^{2}=\frac{4}{\pi} L^{2}\left(\frac{\Delta E_{s} / \tau}{d E_{\tau} / d \sqrt{\tau}}\right)^{2}
$$

where $m(\mathrm{~g})$ is mass loading, $V_{m}\left(\mathrm{~cm}^{3} \mathrm{~mol}^{-1}\right)$ is molar volume of the electrode, $M\left(\mathrm{~g} \mathrm{~mol}^{-1}\right)$ is molar weight of the electrode, $A\left(\mathrm{~cm}^{2}\right)$ is electroactive area of the electrode, $\Delta E_{s}(\mathrm{~V})$ is the change of quasi-equilibrium potential after two sequential relaxation period, $\tau(\mathrm{s})$ is charge or discharge time during each titration, $\mathrm{d} E_{\tau} / \mathrm{d} V \tau\left(\mathrm{V} \mathrm{s}^{-1 / 2}\right)$ is potential shift rate, and $L(\mathrm{~cm})$ is thickness of the electrode. For an electrode-level analysis, the geometric area of the electrode is thus used as the electroactive area to highlight the significance of porosity for the apparent $\mathrm{Li}^{+}$diffusion. 
The above Eq. (S5) can be simplified by applying the small current density for a sufficiently short time in each titration, so that $\mathrm{d} E_{\tau} / \mathrm{d} V \tau$ can be estimated as a stepwise constant for each titration (Figure S19b and S19e):

$$
D=\frac{4}{\pi} L^{2}\left(\frac{\Delta E_{s} / \tau}{\Delta E_{\tau} / \sqrt{\tau}}\right)^{2}=\frac{4 L^{2}}{\pi \tau}\left(\frac{\Delta E_{s}}{\Delta E_{\tau}}\right)^{2}
$$


(a)

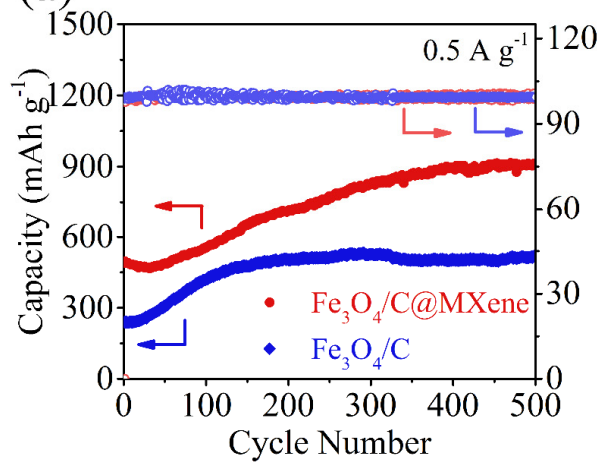

(b)

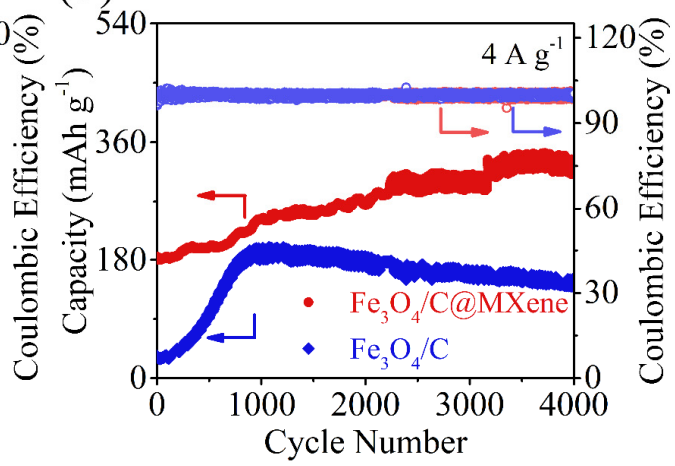

Figure S20. Cycling stability and coulombic efficiency of $\mathrm{Fe}_{3} \mathrm{O}_{4} / \mathrm{C} @$ MXene and $\mathrm{Fe}_{3} \mathrm{O}_{4} / \mathrm{C}$ in halfcell configuration (0.01-3.0 V vs. Li/Li ${ }^{+}$): (a) $0.5 \mathrm{~A} \mathrm{~g}^{-1}$ for $500 \mathrm{GCD}$ cycles; (b) $4 \mathrm{~A} \mathrm{~g}^{-1}$ for 4000 GCD cycles.

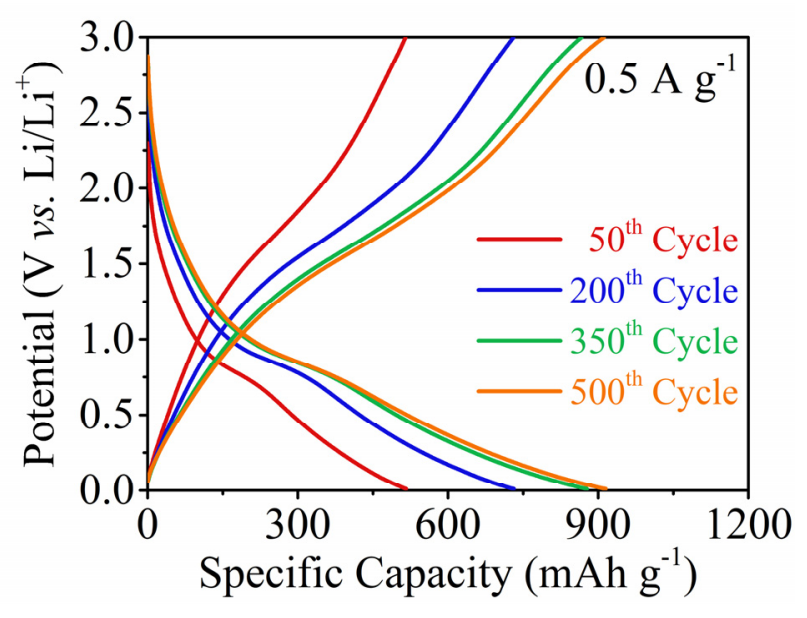

Figure S21. GCD curves of $\mathrm{Fe}_{3} \mathrm{O}_{4} / \mathrm{C} @ \mathrm{MX}$ ene electrode in half-cell configuration (0.01-3.0 V vs. $\left.\mathrm{Li} / \mathrm{Li}^{+}\right)$at $50^{\text {th }}, 200^{\text {th }}, 350^{\text {th }}$, and $500^{\text {th }}$ cycle, respectively. 


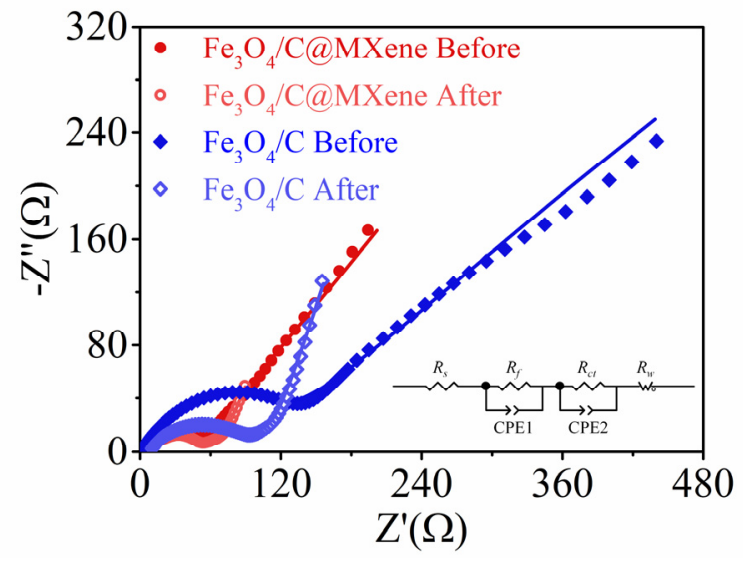

Figure S22. EIS analysis before and after cycling stability test. Inset the circuit model used for impedance fitting.

Table S3. EIS fitting results from Figure S22. $R_{f}$ is thin-film resistance, $R_{c t}$ is charge transfer resistance, and $R_{w}$ is Warburg resistance for ion diffusion.

\begin{tabular}{cccc}
\hline Electrodes & $\boldsymbol{R}_{\boldsymbol{f}}(\boldsymbol{\Omega})$ & $\boldsymbol{R}_{c t}(\boldsymbol{\Omega})$ & $\boldsymbol{R}_{\boldsymbol{w}}(\boldsymbol{\Omega})$ \\
\hline $\mathrm{Fe}_{3} \mathrm{O}_{4} / \mathrm{C} @ M$ Mene (Before Cycling) & 7.7 & 33.6 & 1157 \\
$\mathrm{Fe}_{3} \mathrm{O}_{4} / \mathrm{C} @ \mathrm{MXene}$ (After Cycling) & 1.9 & 38.7 & 66.0 \\
$\mathrm{Fe}_{3} \mathrm{O}_{4} / \mathrm{C}$ (Before Cycling) & 11.7 & 84.3 & 1416 \\
$\mathrm{Fe}_{3} \mathrm{O}_{4} / \mathrm{C}$ (After Cycling) & 14.4 & 59.1 & 87.5 \\
\hline
\end{tabular}



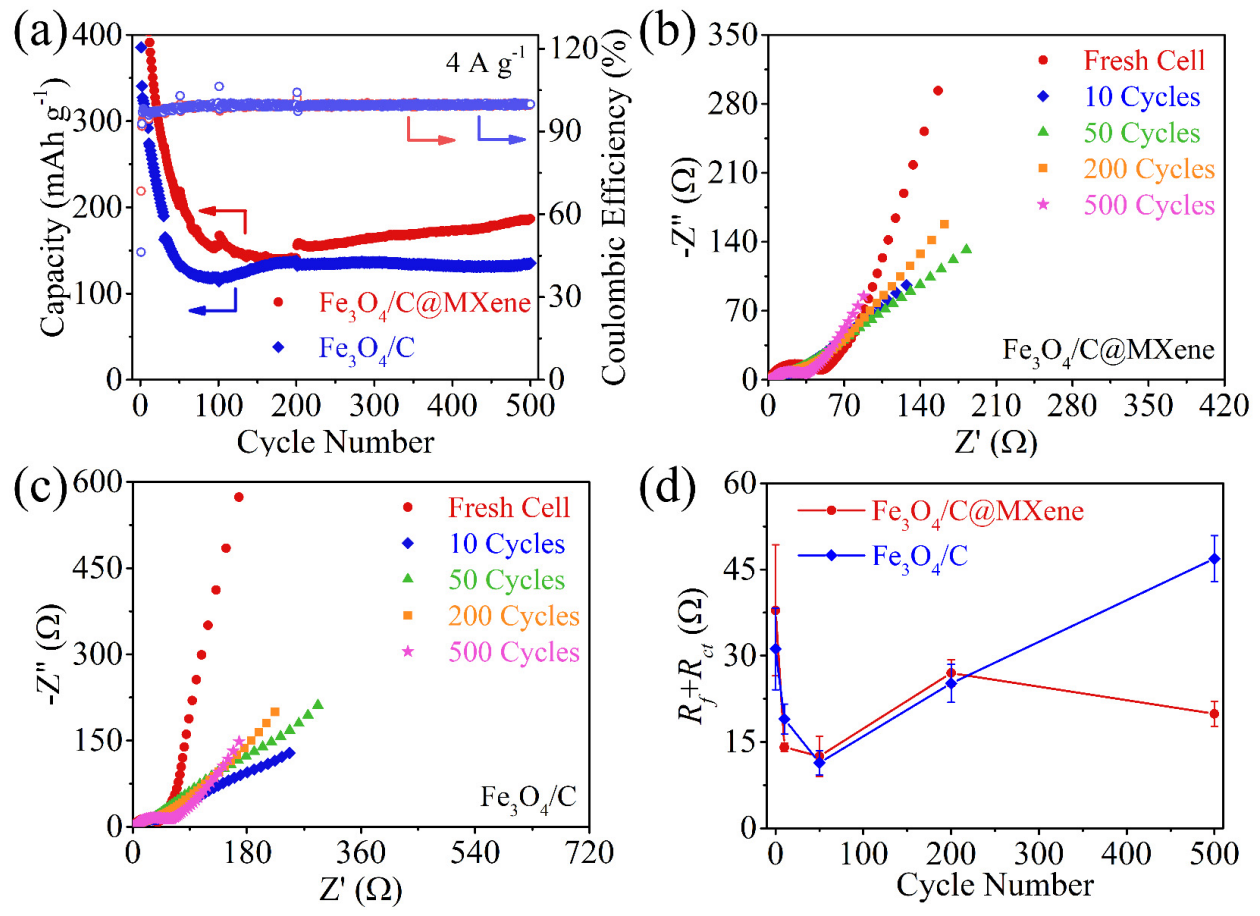

Figure S23. EIS analysis of fresh half-cells before and after cycling stability test. (a) Cycling stability and coulombic efficiency of $\mathrm{Fe}_{3} \mathrm{O}_{4} / \mathrm{C} @$ MXene and pristine $\mathrm{Fe}_{3} \mathrm{O}_{4} / \mathrm{C}_{\text {at }} 4 \mathrm{~A} \mathrm{~g}^{-1}$ for 500 GCD cycles. (b) EIS spectra of $\mathrm{Fe}_{3} \mathrm{O}_{4} / \mathrm{C} @ M X e n e$ after different cycles. (c) EIS spectra of $\mathrm{Fe}_{3} \mathrm{O}_{4} / \mathrm{C}$ after different cycles. (d) Comparison of " $R_{f}+R_{c t}$ " values with cycles. 

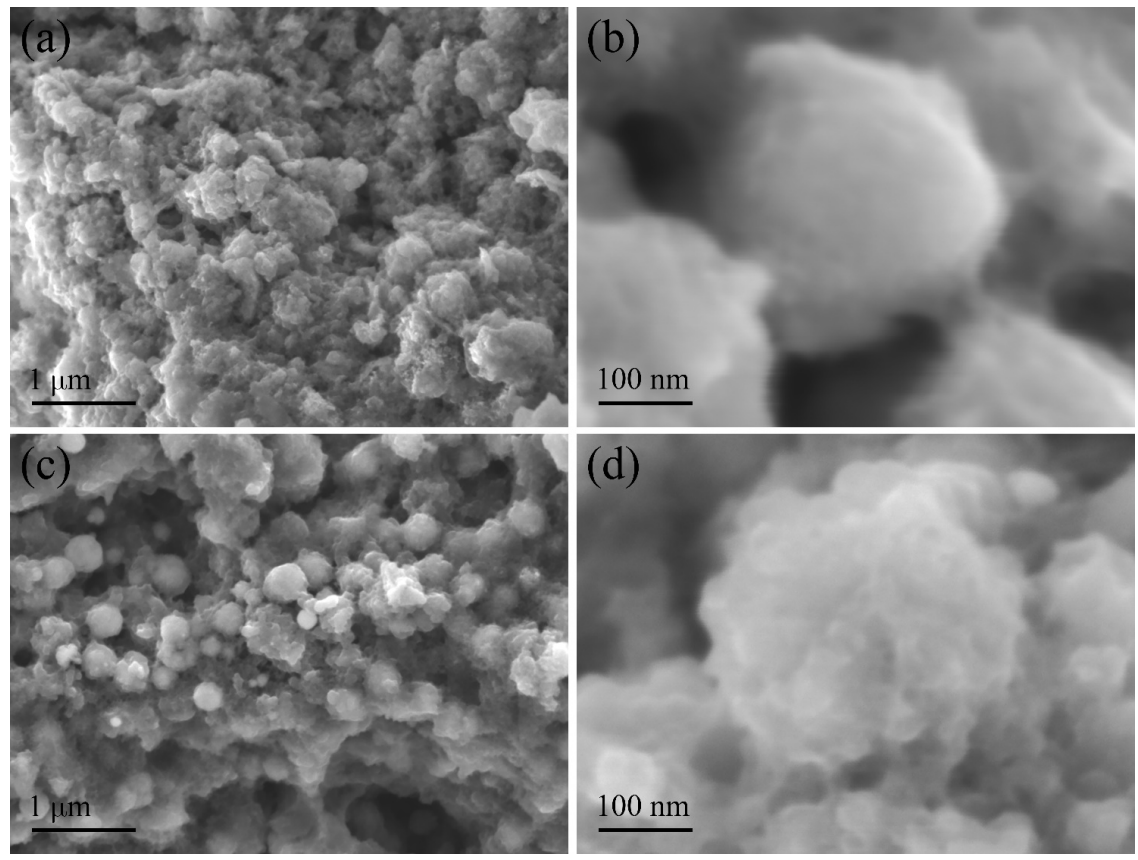

Figure S24. SEM images of (a-b) $\mathrm{Fe}_{3} \mathrm{O}_{4} / \mathrm{C} @$ MXene and (c-d) $\mathrm{Fe}_{3} \mathrm{O}_{4} / \mathrm{C}$ after cycling stability test.
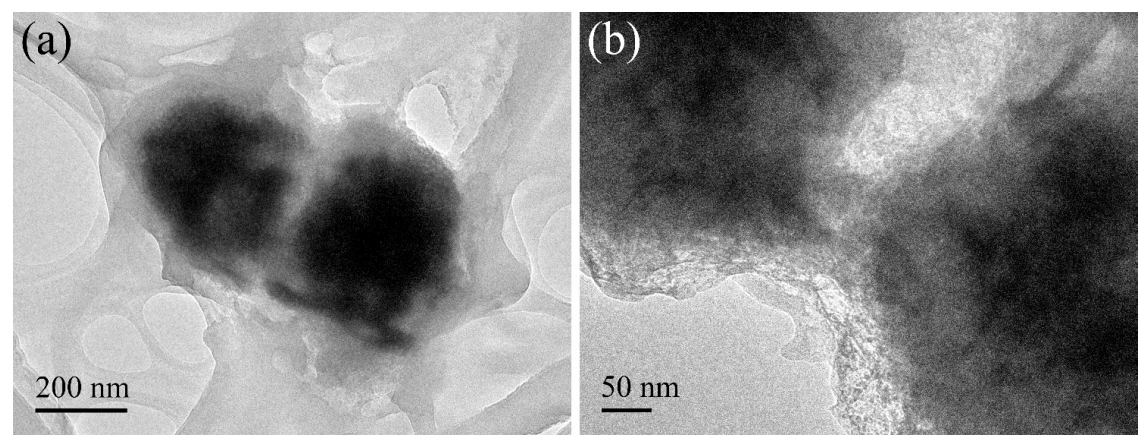

Figure S25. TEM images of $\mathrm{Fe}_{3} \mathrm{O}_{4} / \mathrm{C} @ M X$ Mene after cycling stability test. (a) Low-magnification image. (b) High-magnification image showing nanosheet-like structure wrapping microspheres. 
Table S4. Comparison of electrochemical performance among different anode materials after cycling stability tests.

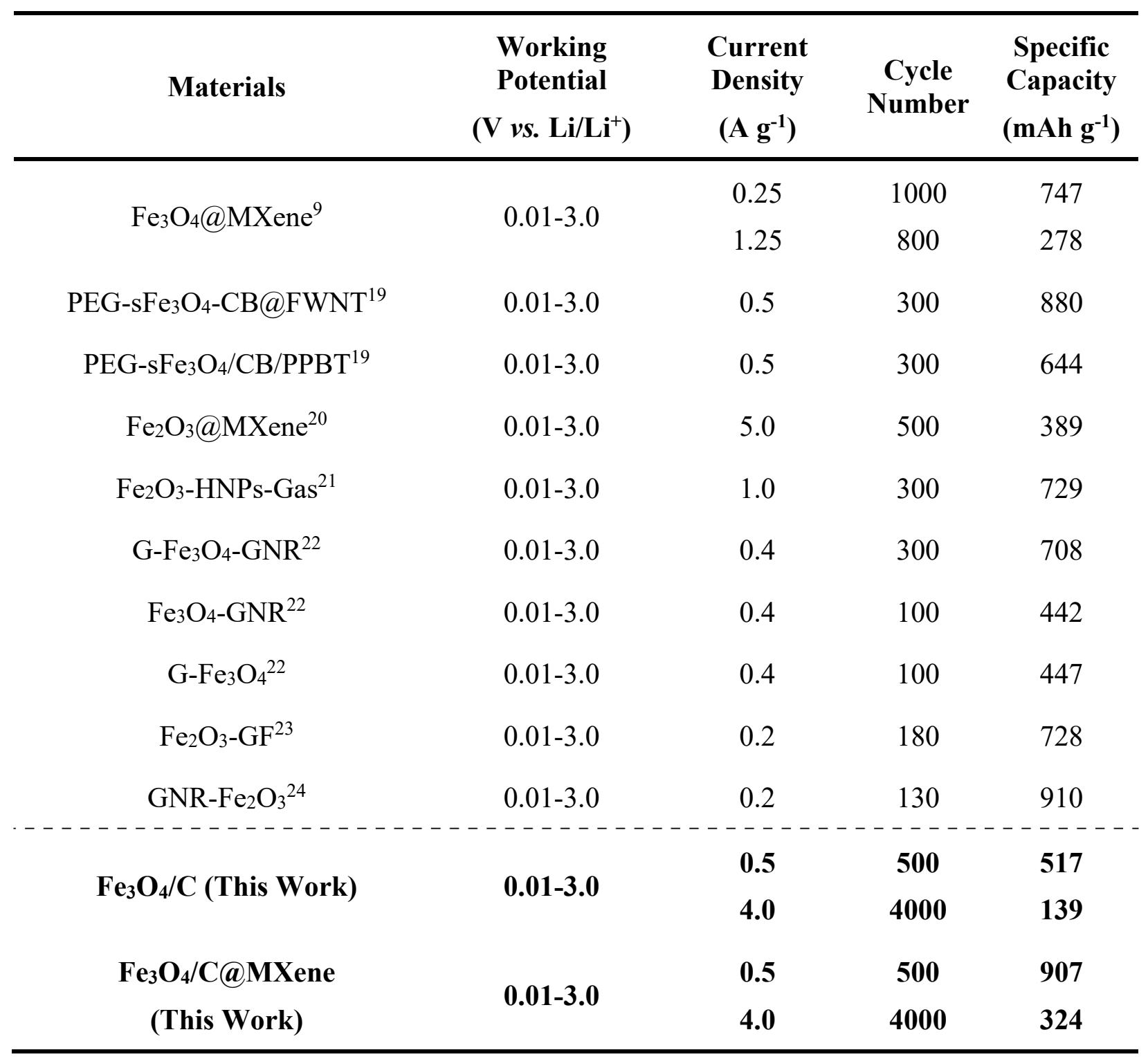



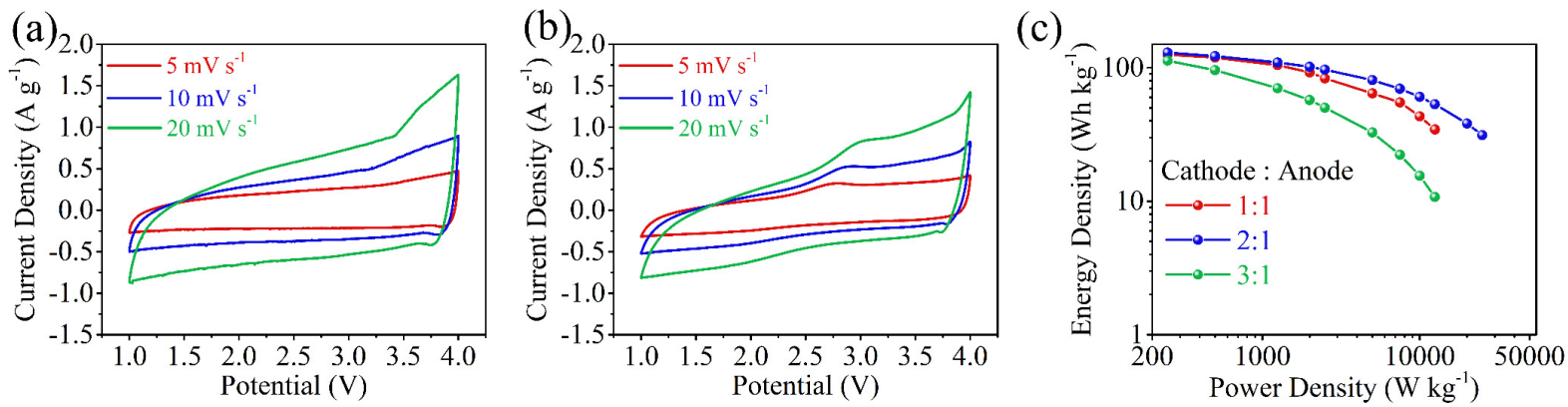

Figure S26. Determination of optimized cathode (i.e., PDPC) to anode (i.e., $\mathrm{Fe}_{3} \mathrm{O}_{4} / \mathrm{C} @ M$ Mene)

mass ratio. (a) CV curves at 5,10, and $20 \mathrm{mV} \mathrm{s}^{-1}$ with mass ratio 1:1. (b) CV curves at 5, 10, and $20 \mathrm{mV} \mathrm{s}^{-1}$ with mass ratio 3:1. (c) Ragone plots comparing the energy and power densities. 


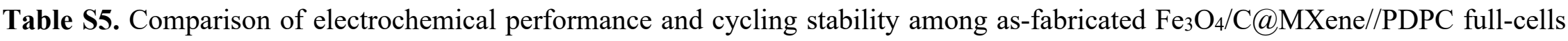
and state-of-the-art full-cell LICs.

\begin{tabular}{|c|c|c|c|c|c|c|c|c|}
\hline \multirow[b]{2}{*}{ Materials } & \multirow[b]{2}{*}{$\begin{array}{c}\text { Working } \\
\text { Potential } \\
\text { (V) }\end{array}$} & \multicolumn{2}{|c|}{ Best Performance } & \multicolumn{2}{|c|}{ Rate Capability } & \multicolumn{3}{|c|}{ Cycling Stability } \\
\hline & & $\begin{array}{c}\text { Energy } \\
\text { Density } \\
\left(\mathrm{Wh} \mathrm{kg}^{-1}\right)\end{array}$ & 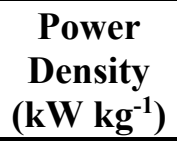 & $\begin{array}{c}\text { Energy } \\
\text { Density } \\
\left(\mathrm{Wh} \mathrm{kg}^{-1}\right)\end{array}$ & $\begin{array}{c}\text { Power } \\
\text { Density } \\
\left(\mathrm{kW} \mathrm{kg}^{-1}\right)\end{array}$ & $\begin{array}{c}\text { Current } \\
\text { Density } \\
\left(\mathrm{A} \mathrm{g}^{-1}\right)\end{array}$ & $\begin{array}{c}\text { Cycle } \\
\text { Number }\end{array}$ & $\begin{array}{c}\text { Capacity } \\
\text { Retention } \\
(\%)\end{array}$ \\
\hline $\mathrm{LTSO}-\mathrm{C} / / \mathrm{AC}^{25}$ & $1.5-4.2$ & 106 & 0.29 & 25 & 28.5 & 2.0 & 2000 & $91.7 \%$ \\
\hline $\mathrm{Nb}_{2} \mathrm{O}_{5} / / \mathrm{AC}^{26}$ & $1.0-3.5$ & 96 & 0.19 & 65 & 5.4 & 2.2 & 1000 & $87.0 \%$ \\
\hline $\mathrm{AC} / / \mathrm{LFV}-\mathrm{GC}^{27}$ & $1.0-4.0$ & 126 & 0.11 & 77 & 3.4 & 1.0 & 1000 & $74.0 \%$ \\
\hline $\mathrm{Co}_{3} \mathrm{ZnC} @ \mathrm{NC} / / \mathrm{AC}^{28}$ & $1.0-4.5$ & 141 & 0.28 & 15 & 10.3 & 1.0 & 1000 & $80.0 \%$ \\
\hline HDMPC//HDMPC 29 & $1.0-4.0$ & 106 & 0.50 & 20 & 50.0 & 2.0 & 8000 & $88.3 \%$ \\
\hline Graphite//AC 30 & $2.2-4.0$ & 55 & 0.20 & 20 & 3.0 & 0.5 & 15000 & $>95 \%$ \\
\hline STO-C//AC 31 & $1.0-4.2$ & 98 & 0.26 & 33 & 26.0 & 5.0 & 10000 & $85.8 \%$ \\
\hline $\mathrm{BiVO}_{4} / / \mathrm{PRGO}^{32}$ & $0.01-4.0$ & 152 & 0.38 & 20 & 10.0 & 0.9 & 6000 & $81.0 \%$ \\
\hline $\mathrm{MnO} @ \mathrm{C} / / \mathrm{AC}^{33}$ & $0.1-4.0$ & 118 & 0.41 & 28 & 10.3 & 1.0 & 3000 & $76.0 \%$ \\
\hline $\mathrm{AMC} / / \mathrm{AC}^{34}$ & $0.5-4.0$ & 133 & 0.21 & 42 & 11.2 & 5.0 & 5000 & $81.8 \%$ \\
\hline $\mathrm{Li}_{3} \mathrm{VO}_{4} / / \mathrm{AC}^{35}$ & $1.0-4.0$ & 136 & 0.53 & 24 & 11.0 & 2.0 & 1500 & $87.0 \%$ \\
\hline $\mathrm{GCNT} / / \mathrm{GCNT}^{36}$ & $0.01-4.3$ & 120 & 0.10 & 29 & 20.5 & 1.0 & 10000 & $89.0 \%$ \\
\hline $\mathrm{MnNCN} / / \mathrm{AC}^{37}$ & $0.1-4.0$ & 103 & 0.13 & 10 & 8.5 & 5.0 & 5000 & $>95 \%$ \\
\hline $\mathrm{LTO} / / \mathrm{AC}^{38}$ & $1.5-3.0$ & 52 & 0.22 & 13 & 57.6 & 25.0 & 2000 & $97.0 \%$ \\
\hline MXene//LNMCO 39 & $0.5-4.0$ & 160 & 0.22 & 25 & 4.4 & 0.3 & 100 & $84.0 \%$ \\
\hline $\mathrm{MnO}-\mathrm{C} / / \mathrm{CNS}^{40}$ & $1.0-4.0$ & 83 & 0.17 & 21 & 32.0 & 5.0 & 5000 & $70.0 \%$ \\
\hline $\mathrm{GCY} / / \mathrm{AC}^{41}$ & $2.0-4.0$ & 111 & 0.10 & 95 & 1.0 & 0.2 & 1000 & $94.7 \%$ \\
\hline $\mathrm{TiC} / / \mathrm{AC}^{42}$ & $0.0-4.5$ & 102 & 0.45 & 26 & 45.0 & 2.0 & 5000 & $82.0 \%$ \\
\hline $\mathrm{rGO} / / \mathrm{rGO}^{43}$ & $0.0-4.2$ & 160 & 1.00 & 50 & 20.0 & 1.8 & 1000 & $90.0 \%$ \\
\hline $\mathrm{MnFe}_{2} \mathrm{O}_{4} / / \mathrm{AC}^{44}$ & $0.0-4.0$ & $\underline{157}$ & 0.20 & 58 & 22.0 & 2.0 & 6000 & $86.5 \%$ \\
\hline $\begin{array}{c}\mathrm{Fe}_{3} \mathrm{O}_{4} / \mathrm{C} @ \text { MXene//PDPC } \\
\text { (This Work) }\end{array}$ & $1.0-4.0$ & 130 & 0.25 & 31 & 25.0 & 1.0 & 5000 & $86.5 \%$ \\
\hline
\end{tabular}




\section{References}

(1) Anasori, B.; Lukatskaya, M. R.; Gogotsi, Y. 2D Metal Carbides and Nitrides (MXenes) for Energy Storage. Nat. Rev. Mater. 2017, 2 (2), 16098.

(2) Xie, X.; Zhao, M.-Q.; Anasori, B.; Maleski, K.; Ren, C. E.; Li, J.; Byles, B. W.; Pomerantseva, E.; Wang, G.; Gogotsi, Y. Porous Heterostructured MXene/Carbon Nanotube Composite Paper with High Volumetric Capacity for Sodium-Based Energy Storage Devices. Nano Energy 2016, 26, 513-523.

(3) Sun, Z.-X.; Su, F.-W.; Forsling, W.; Samskog, P.-O. Surface Characteristics of Magnetite in Aqueous Suspension. J. Colloid Interface Sci. 1998, 197 (1), 151-159.

(4) Wang, S.; Wang, R.; Zhang, Y.; Zhang, L. Highly Porous Carbon with Large Electrochemical Ion Absorption Capability for High-Performance Supercapacitors and Ion Capacitors. Nanotechnology 2017, 28 (44), 445406.

(5) Wang, S.; Wang, R.; Zhang, Y.; Jin, D.; Zhang, L. Scalable and Sustainable Synthesis of Carbon Microspheres via a Purification-Free Strategy for Sodium-Ion Capacitors. J. Power Sources 2018, 379, 33-40.

(6) Cançado, L. G.; Takai, K.; Enoki, T.; Endo, M.; Kim, Y. A.; Mizusaki, H.; Jorio, A.; Coelho, L. N.; Magalhães-Paniago, R.; Pimenta, M. A. General Equation for the Determination of the Crystallite Size $L_{a}$ of Nanographite by Raman Spectroscopy. Appl. Phys. Lett. 2006, 88 (16), 163106.

(7) Ali, A.; Hantanasirisakul, K.; Abdala, A.; Urbankowski, P.; Zhao, M.-Q.; Anasori, B.; Gogotsi, Y.; Aïssa, B.; Mahmoud, K. A. Effect of Synthesis on Performance of MXene/Iron Oxide Anode Material for Lithium-Ion Batteries. Langmuir 2018, 34 (38), 11325-11334.

(8) Kong, F.; He, X.; Liu, Q.; Qi, X.; Sun, D.; Zheng, Y.; Wang, R.; Bai, Y. Further Surface Modification by Carbon Coating for In-Situ Growth of $\mathrm{Fe}_{3} \mathrm{O}_{4}$ Nanoparticles on MXene $\mathrm{Ti}_{3} \mathrm{C}_{2}$ Multilayers for Advanced Li-Ion Storage. Electrochim. Acta 2018, 289, 228-237.

(9) Wang, Y.; Li, Y.; Qiu, Z.; Wu, X.; Zhou, P.; Zhou, T.; Zhao, J.; Miao, Z.; Zhou, J.; Zhuo, $\mathrm{S} . \mathrm{Fe}_{3} \mathrm{O}_{4} @ \mathrm{Ti}_{3} \mathrm{C}_{2} \mathrm{MXene}$ Hybrids with Ultrahigh Volumetric Capacity as an Anode Material for Lithium-Ion Batteries. J. Mater. Chem. A 2018, 6 (24), 11189-11197.

(10) Zhang, Y.-J.; Qu, J.; Hao, S.-M.; Chang, W.; Ji, Q.-Y.; Yu, Z.-Z. High Lithium Storage Capacity and Long Cycling Life $\mathrm{Fe}_{3} \mathrm{~S}_{4}$ Anodes with Reversible Solid Electrolyte Interface Films and Sandwiched Reduced Graphene Oxide Shells. ACS Appl. Mater. Interfaces 2017, 9 (48), 41878-41886.

(11) Pan, L.; Zhu, X.-D.; Xie, X.-M.; Liu, Y.-T. Smart Hybridization of $\mathrm{TiO}_{2}$ Nanorods and $\mathrm{Fe}_{3} \mathrm{O}_{4}$ Nanoparticles with Pristine Graphene Nanosheets: Hierarchically Nanoengineered Ternary Heterostructures for High-Rate Lithium Storage. Adv. Funct. Mater. 2015, 25 (22), 3341-3350.

(12) Zhao, M.-Q.; Torelli, M.; Ren, C. E.; Ghidiu, M.; Ling, Z.; Anasori, B.; Barsoum, M. W.; Gogotsi, Y. 2D Titanium Carbide and Transition Metal Oxides Hybrid Electrodes for LiIon Storage. Nano Energy 2016, 30, 603-613. 
(13) Liu, Y.-T.; Zhang, P.; Sun, N.; Anasori, B.; Zhu, Q.-Z.; Liu, H.; Gogotsi, Y.; Xu, B. SelfAssembly of Transition Metal Oxide Nanostructures on MXene Nanosheets for Fast and Stable Lithium Storage. Adv. Mater. 2018, 30 (23), 1707334.

(14) Ma, Z.; Zhou, X.; Deng, W.; Lei, D.; Liu, Z. 3D Porous MXene $\left(\mathrm{Ti}_{3} \mathrm{C}_{2}\right) /$ Reduced Graphene Oxide Hybrid Films for Advanced Lithium Storage. ACS Appl. Mater. Interfaces 2018, 10 (4), 3634-3643.

(15) Chen, C.; Xie, X.; Anasori, B.; Sarycheva, A.; Makaryan, T.; Zhao, M.; Urbankowski, P.; Miao, L.; Jiang, J.; Gogotsi, Y. MoS2-on-MXene Heterostructures as Highly Reversible Anode Materials for Lithium-Ion Batteries. Angew. Chemie Int. Ed. 2018, 57 (7), 18461850.

(16) Yang, X.; Rogach, A. L. Electrochemical Techniques in Battery Research: A Tutorial for Nonelectrochemists. Adv. Energy Mater. 2019, 9 (25), 1900747.

(17) Weppner, W. Determination of the Kinetic Parameters of Mixed-Conducting Electrodes and Application to the System Li3Sb. J. Electrochem. Soc. 1977, 124 (10), 1569-1578.

(18) Dees, D. W.; Kawauchi, S.; Abraham, D. P.; Prakash, J. Analysis of the Galvanostatic Intermittent Titration Technique (GITT) as Applied to a Lithium-Ion Porous Electrode. $J$. Power Sources 2009, 189 (1), 263-268.

(19) Kwon, Y. H.; Park, J. J.; Housel, L. M.; Minnici, K.; Zhang, G.; Lee, S. R.; Lee, S. W.; Chen, Z.; Noda, S.; Takeuchi, E. S.; Takeuchi, K. J.; Marschilok, A. C.; Reichmanis, E. Carbon Nanotube Web with Carboxylated Polythiophene "Assist" for High-Performance Battery Electrodes. ACS Nano 2018, 12 (4), 3126-3139.

(20) Tang, X.; Liu, H.; Guo, X.; Wang, S.; Wu, W.; Mondal, A. K.; Wang, C.; Wang, G. A Novel Lithium-Ion Hybrid Capacitor Based on an Aerogel-like MXene Wrapped $\mathrm{Fe}_{2} \mathrm{O}_{3}$ Nanosphere Anode and a 3D Nitrogen Sulphur Dual-Doped Porous Carbon Cathode. Mater. Chem. Front. 2018, 2 (10), 1811-1821.

(21) Liu, L.; Yang, X.; Lv, C.; Zhu, A.; Zhu, X.; Guo, S.; Chen, C.; Yang, D. Seaweed-Derived Route to $\mathrm{Fe}_{2} \mathrm{O}_{3}$ Hollow Nanoparticles/N-Doped Graphene Aerogels with High Lithium Ion Storage Performance. ACS Appl. Mater. Interfaces 2016, 8 (11), 7047-7053.

(22) Li, L.; Kovalchuk, A.; Fei, H.; Peng, Z.; Li, Y.; Kim, N. D.; Xiang, C.; Yang, Y.; Ruan, G.; Tour, J. M. Enhanced Cycling Stability of Lithium-Ion Batteries Using Graphene-Wrapped $\mathrm{Fe}_{3} \mathrm{O}_{4}$-Graphene Nanoribbons as Anode Materials. Adv. Energy Mater. 2015, 5 (14), 1500171.

(23) Chen, M.; Liu, J.; Chao, D.; Wang, J.; Yin, J.; Lin, J.; Jin Fan, H.; Xiang Shen, Z. Porous $\alpha-\mathrm{Fe}_{2} \mathrm{O}_{3}$ Nanorods Supported on Carbon Nanotubes-Graphene Foam as Superior Anode for Lithium Ion Batteries. Nano Energy 2014, 9, 364-372.

(24) Lin, J.; Raji, A.-R. O.; Nan, K.; Peng, Z.; Yan, Z.; Samuel, E. L. G.; Natelson, D.; Tour, J. M. Iron Oxide Nanoparticle and Graphene Nanoribbon Composite as an Anode Material for High-Performance Li-Ion Batteries. Adv. Funct. Mater. 2014, 24 (14), 2044-2048.

(25) Wang, S.; Wang, R.; Bian, Y.; Jin, D.; Zhang, Y.; Zhang, L. In-Situ Encapsulation of Pseudocapacitive $\mathrm{Li}_{2} \mathrm{TiSiO}_{5}$ Nanoparticles into Fibrous Carbon Framework for Ultrafast and Stable Lithium Storage. Nano Energy 2019, 55, 173-181. 
(26) Deng, B. H.; Lei, T. Y.; Zhu, W. H.; Xiao, L.; Liu, J. P. In-Plane Assembled Orthorhombic $\mathrm{Nb}_{2} \mathrm{O}_{5}$ Nanorod Films with High-Rate $\mathrm{Li}^{+}$Intercalation for High-Performance Flexible LiIon Capacitors. Adv. Funct. Mater. 2018, 28 (1), 1704330.

(27) Zhang, Y.; Zhang, Z.; Tang, Y.; Jia, D.; Huang, Y.; Pang, W.; Guo, Z.; Zhou, Z. LiFePO4 Particles Embedded in Fast Bifunctional Conductor RGO\&C@ $\mathrm{Li}_{3} \mathrm{~V}_{2}\left(\mathrm{PO}_{4}\right)_{3}$ Nanosheets as Cathodes for High-Performance Li-Ion Hybrid Capacitors. Adv. Funct. Mater. 2019, 29 (17), 1807895.

(28) Zhu, G.; Chen, T.; Wang, L.; Ma, L.; Hu, Y.; Chen, R.; Wang, Y.; Wang, C.; Yan, W.; Tie, Z.; Liu, J.; Jin, Z. High Energy Density Hybrid Lithium-Ion Capacitor Enabled by Co3ZnC@N-Doped Carbon Nanopolyhedra Anode and Microporous Carbon Cathode. Energy Storage Mater. 2018, 14, 246-252.

(29) Niu, J.; Shao, R.; Liu, M.; Liang, J.; Zhang, Z.; Dou, M.; Huang, Y.; Wang, F. Porous Carbon Electrodes with Battery-Capacitive Storage Features for High Performance Li-Ion Capacitors. Energy Storage Mater. 2018, 12, 145-152.

(30) Jeżowski, P.; Crosnier, O.; Deunf, E.; Poizot, P.; Béguin, F.; Brousse, T. Safe and Recyclable Lithium-Ion Capacitors Using Sacrificial Organic Lithium Salt. Nat. Mater. 2018, 17 (2), 167-173.

(31) Wang, L.; Yang, H.; Shu, T.; Xin, Y.; Chen, X.; Li, Y.; Li, H.; Hu, X. Nanoengineering SDoped $\mathrm{TiO}_{2}$ Embedded Carbon Nanosheets for Pseudocapacitance-Enhanced Li-Ion Capacitors. ACS Appl. Energy Mater. 2018, 1 (4), 1708-1715.

(32) Dubal, D. P.; Jayaramulu, K.; Zboril, R.; Fischer, R. A.; Gomez-Romero, P. Unveiling $\mathrm{BiVO}_{4}$ Nanorods as a Novel Anode Material for High Performance Lithium Ion Capacitors: Beyond Intercalation Strategies. J. Mater. Chem. A 2018, 6 (14), 6096-6106.

(33) Yan, D.; Li, S.-H.; Guo, L.-P.; Dong, X.-L.; Chen, Z.-Y.; Li, W.-C. Hard@Soft Integrated Morning Glory Like Porous Carbon as a Cathode for a High-Energy Lithium Ion Capacitor. ACS Appl. Mater. Interfaces 2018, 10 (50), 43946-43952.

(34) Lee, W. S. V.; Huang, X.; Tan, T. L.; Xue, J. M. Low Li ${ }^{+}$Insertion Barrier Carbon for High Energy Efficient Lithium-Ion Capacitor. ACS Appl. Mater. Interfaces 2018, 10 (2), 16901700 .

(35) Shen, L.; Lv, H.; Chen, S.; Kopold, P.; van Aken, P. A.; Wu, X.; Maier, J.; Yu, Y. Peapodlike $\mathrm{Li}_{3} \mathrm{VO}_{4} / \mathrm{N}$-Doped Carbon Nanowires with Pseudocapacitive Properties as Advanced Materials for High-Energy Lithium-Ion Capacitors. Adv. Mater. 2017, 29 (27), 1700142.

(36) Salvatierra, R. V.; Zakhidov, D.; Sha, J.; Kim, N. D.; Lee, S.-K.; Raji, A.-R. O.; Zhao, N.; Tour, J. M. Graphene Carbon Nanotube Carpets Grown Using Binary Catalysts for HighPerformance Lithium-Ion Capacitors. ACS Nano 2017, 11 (3), 2724-2733.

(37) Liu, C.; Zhang, C.; Fu, H.; Nan, X.; Cao, G. Exploiting High-Performance Anode through Tuning the Character of Chemical Bonds for Li-Ion Batteries and Capacitors. Adv. Energy Mater. 2017, 7 (1), 1601127.

(38) Wang, G.; Lu, C.; Zhang, X.; Wan, B.; Liu, H.; Xia, M.; Gou, H.; Xin, G.; Lian, J.; Zhang, Y. Toward Ultrafast Lithium Ion Capacitors: A Novel Atomic Layer Deposition Seeded Preparation of $\mathrm{Li}_{4} \mathrm{Ti}_{5} \mathrm{O}_{12} /$ Graphene Anode. Nano Energy 2017, 36, 46-57. 
(39) Kajiyama, S.; Szabova, L.; Iinuma, H.; Sugahara, A.; Gotoh, K.; Sodeyama, K.; Tateyama, Y.; Okubo, M.; Yamada, A. Enhanced Li-Ion Accessibility in MXene Titanium Carbide by Steric Chloride Termination. Adv. Energy Mater. 2017, 7 (9), 1601873.

(40) Zhao, Y.; Cui, Y.; Shi, J.; Liu, W.; Shi, Z.; Chen, S.; Wang, X.; Wang, H. Two-Dimensional Biomass-Derived Carbon Nanosheets and $\mathrm{MnO} /$ Carbon Electrodes for High-Performance Li-Ion Capacitors. J. Mater. Chem. A 2017, 5 (29), 15243-15252.

(41) Du, H.; Yang, H.; Huang, C.; He, J.; Liu, H.; Li, Y. Graphdiyne Applied for Lithium-Ion Capacitors Displaying High Power and Energy Densities. Nano Energy 2016, 22, 615-622.

(42) Wang, H.; Zhang, Y.; Ang, H.; Zhang, Y.; Tan, H. T.; Zhang, Y.; Guo, Y.; Franklin, J. B.; Wu, X. L.; Srinivasan, M.; Fan, H. J.; Yan, Q. A High-Energy Lithium-Ion Capacitor by Integration of a 3D Interconnected Titanium Carbide Nanoparticle Chain Anode with a Pyridine-Derived Porous Nitrogen-Doped Carbon Cathode. Adv. Funct. Mater. 2016, 26 (18), 3082-3093.

(43) Shan, X.-Y.; Wang, Y.; Wang, D.-W.; Li, F.; Cheng, H.-M. Armoring Graphene Cathodes for High-Rate and Long-Life Lithium Ion Supercapacitors. Adv. Energy Mater. 2016, 6 (6), 1502064.

(44) Lee, W. S. V.; Peng, E.; Li, M.; Huang, X.; Xue, J. M. Rational Design of Stable 4 V Lithium Ion Capacitor. Nano Energy 2016, 27, 202-212. 